\section{International Scientific Journal Theoretical \& Applied Science}

p-ISSN: 2308-4944 (print) e-ISSN: 2409-0085 (online)

Year: $2015 \quad$ Issue: 08 Volume: 28

Published: $30.08 .2015 \quad$ http://T-Science.org
Gennady Evgenievich Markelov

Candidate of Engineering Sciences, associate professor,

Bauman Moscow State Technical University, Moscow, Russia markelov@bmstu.ru

SECTION 2. Applied mathematics. Mathematical modeling.

\title{
CONSTRUCTING A WORKING MATHEMATICAL MODEL
}

Abstract: The concept of a working mathematical model has been formulated. Features of construction of such mathematical models have been briefly described. An illustrative example of the process of construction of a working mathematical model of the element of a technical system has been discussed. Working mathematical model with the desired properties from the point of view of a particular study is a valuable intellectual product. The use of such a model helps to reduce the time and cost of research and also allows efficient use of mathematical modeling.

Key words: principles of mathematical modeling, properties of mathematical models, working mathematical model.

Language: English

Citation: Markelov GE (2015) CONSTRUCTING A WORKING MATHEMATICAL MODEL. ISJ Theoretical \& Applied Science 08 (28): 44-46.

Soi: http://s-0-i.org/1.1/TAS-08-28-6 Doi: crossef http://dx.doi.org/10.15863/TAS.2015.08.28.6

\section{Introduction}

As a rule, mathematical modeling of technical objects includes the following basic steps.

The first phase of mathematical modeling involves an informal transition from the object of research to its conceptual model. Conceptual model is a conventional description of the object of research. Such a description should include the features and quantitative characteristics of the object that are important from the point of view of the case being analyzed. At the same time, it is necessary to justify the assumptions and simplifications that allow not taking into account the features and quantitative characteristics of the object of research that are considered immaterial.

The second phase is the mathematical description of the conceptual model. Using the formal description, one or more mathematical models of the object of research can be constructed.

The third phase of mathematical modeling involves qualitative and estimated quantitative analysis of the mathematical model. Sometimes the results of analysis may lead to the need to clarify or revise the conceptual model (that is, it will be necessary to re-implement the first phase of mathematical modeling). To make an informed choice of a model for the subsequent detailed quantitative analysis, it is necessary to compare the results of analysis of various mathematical models. As a result of this phase, a working mathematical model is constructed, that is, a model for detailed quantitative analysis.

During the fourth phase, a computational problem is formulated.

In the fifth phase of mathematical modeling, it is necessary to make an informed choice or create a numerical method. As a rule, the resulting numerical method does not include many of the necessary details without which it is impossible to use computer technology. To obtain an implementable algorithm for the computing experiment, a detailed specification of all necessary steps is needed. An effective algorithm for the computing experiment is created during the sixth phase of mathematical modeling.

The seventh phase involves the development of software that implements the computational algorithm.

The software product is tested during the eighth phase. Sometimes, a thorough check of the results of calculations can detect deficiencies that require reimplementation of the previous phases of mathematical modeling. After eliminating all the deficiencies, implementation of the computational experiment can be started. Computational experiment is the ninth and final phase of mathematical modeling.

Possibilities of mathematical modeling that are described in detail in educational and scientific literature (for example, [1-5]) are often used 
inefficiently. The reason for this is that the mathematical model of the object of research does not have the desired properties.

The purpose of this paper is to describe the features that allow constructing a working mathematical model with the desired properties from the point of view of a particular study. The set of properties may include, for example, the completeness, accuracy, adequacy, productivity, economy, robustness, as well as other properties $[2 ; 5]$. Obviously, such a mathematical model is a valuable intellectual product that is, within the research scope, equivalent to the object of research.

\section{Unified approach to the construction of a mathematical model}

To construct a working mathematical model with the desired properties from the point of view of a particular research project, it is often necessary to fulfill contradictory requirements. Such requirements can only be met with the help of a reasonable compromise that largely depends on the level of training of the researcher.

Compromise is made possible by following the rules and recommendations summarizing practical experience in the development of mathematical models. In that context, the principles from [6] are of particular interest. Thanks to the wise use of these principles, it is possible to implement a unified approach to the construction of a mathematical model with the desired properties from the point of view of a particular research project. For example, paper [7] contains a description of features of the process of constructing a mathematical model with the desired properties from the point of view of the study some results of which are described in [8-10].

\section{Working mathematical model of the element of a technical system}

Let us discuss an illustrative example of the process of construction of a working mathematical model.

\subsection{Statement of the problem}

Let us assume that the following value is of interest in the conducted research:

$$
z=x / r(y)
$$

where $r(y)=r_{0}\left[1+\beta\left(y-y_{0}\right)\right]$ is the function of a real variable $y ; y=y(t)$ is the time function $t$, where $y\left(t_{0}\right)=y_{0} ; y_{0}$ and $t_{0}$ are known non-negative values; $x, r_{0}$ and $\beta$ are known positive constants. In this case, there may be a steady state, for which the following equation holds true:

$$
x^{2} / r(\bar{y})=1+\sigma\left(\bar{y}-y_{0}\right),
$$

where $\bar{y}$ is the steady-state value, and $y_{0} \leq y \leq \bar{y} ; \sigma$ is a known constant.
Here $z$ is the sought quantity that can characterize the potential or flow of a physical substance in the element of the technical system. Let us construct a working mathematical model of the object of research. The model must be sufficiently complete, accurate, adequate, productive and simple.

\subsection{Solution}

To solve this problem, let us create a hierarchy of mathematical models of the object of research and define the conditions under which it is possible to find the sought quantity with a relative error of not more than the allowable limit $\delta_{0}$.

If the difference $y-y_{0}$ is rather small, then we can use (1) to find the sought quantity according to the following formula:

$$
z_{0}=x / r_{0}
$$

Let us define the conditions under which the resulting formula is applicable. To do this, let us analyze the steady state.

We can use (2) to find

$$
\bar{y}=y_{0}+\frac{1}{2 \beta}\left(-1+\sqrt{1+4 \beta x^{2} /\left(\sigma r_{0}\right)}\right),
$$

and then define the steady-state value

$$
\bar{z}=x / r(\bar{y})=2 z_{0} /\left(1+\sqrt{1+4 \beta x^{2} /\left(\sigma r_{0}\right)}\right) .
$$

It is evident that $\bar{z} \leq z \leq z_{0}$. Then the following holds true for the relative error of $z_{0}$ :

$$
\delta\left(z_{0}\right)=\left|z-z_{0}\right| /|z|=z_{0} / z-1 \leq z_{0} / \bar{z}-1 .
$$

Thus, if the condition

$$
z_{0} / \bar{z}-1 \leq \delta_{0}
$$

is met, we can use the formula (3) to find the sought quantity with a relative error of not more than $\delta_{0}$. Then we arrive at the inequality

$$
\beta x^{2} /\left(\sigma r_{0}\right) \leq \delta_{0}^{2}+\delta_{0},
$$

subject to which the mathematical model (3) is sufficiently complete, accurate, adequate, productive and simple; and it is reasonable to use the formula (3) to find the sought quantity.

If a non-steady state can be analyzed, it is possible to determine the conditions under which the mathematical model (4) is applicable.

Let us suppose the following first order ordinary differential equation is valid for the object of research:

$$
c(y) \frac{d y}{d t}=x^{2} / r(y)-\sigma\left(y-y_{0}\right),
$$

where $c(y)=c_{0}\left[1+\gamma\left(y-y_{0}\right)\right]$ is the function of a real variable $y ; c_{0}$ and $\gamma$ are known positive constants. Then, according to (1) and (6), let us formulate a Cauchy problem 


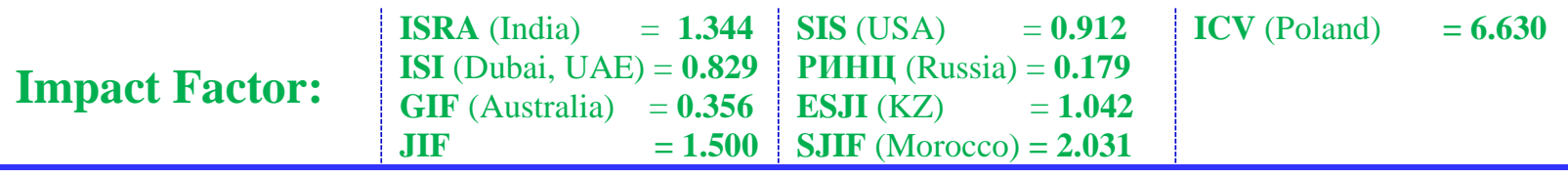

$$
\begin{aligned}
& c_{0}\left[\gamma\left(z_{0}-z\right)+\beta z\right] z_{0} \frac{d z}{d t}=\beta\left[\sigma\left(z_{0}-z\right)-x \beta z^{2}\right] z^{2}, \\
& z\left(t_{0}\right)=z_{0} .
\end{aligned}
$$

Thus, if the condition

$$
\delta(\bar{z})=|z-\bar{z}| /|z|=1-\bar{z} / z \leq \delta_{0}
$$

is met, we can use the formula (4) to find the sought quantity with a relative error of not more than $\delta_{0}$, where $\delta_{0}<z_{0} / \bar{z}-1$, since otherwise the formula (3) is to be used. Then let us find a time point

$$
\begin{gathered}
t_{1}=t_{0}+c_{0}\left[z_{0}\left(\gamma\left(\bar{z}-z_{0}\right)-\beta \bar{z}\right) \ln \left(\delta_{0} z_{0} /\left(z_{0}-\bar{z}\right)\right)+\right. \\
+\left(z_{0} \beta \bar{z}+\gamma\left(z_{0}-\bar{z}\right) z_{0}-\right. \\
\left.-\beta\left(2 z_{0}-\bar{z}\right) \bar{z}\right) \ln \left(2-\delta_{0}-\bar{z} / z_{0}\right)+ \\
\left.+\gamma z_{0}\left(2 z_{0}-\bar{z}\right)\left(\delta_{0}-1+\bar{z} / z_{0}\right)\right]\left(\sigma \beta\left(2 z_{0}-\bar{z}\right) \bar{z}\right)^{-1},
\end{gathered}
$$

for which $z\left(t_{1}\right)=\bar{z} /\left(1-\delta_{0}\right)$. In this case, according to (7), the steady-state value $\bar{z}$ can be considered equal to $z(t)$ at $t \geq t_{1}$ with a relative error of not more than $\delta_{0}$.

If the inequality (5) is not true, then the mathematical model (4) is sufficiently complete, accurate, adequate, productive and simple at $t \geq t_{1}$; and it is reasonable to use the formula (4) to find the sought quantity.

\subsection{Results}

By creating a hierarchy of mathematical models, it is possible to construct a working mathematical model with the desired properties from the point of view of a particular research project. Indeed, if the inequality (5) holds true, then the mathematical model (3) is considered to be a working mathematical model, otherwise, it is necessary to determine whether it is possible not to consider the time interval from $t_{0}$ to $t_{1}$ in the conducted research. If this is possible, the mathematical model (4) is selected as the working one; otherwise, (7) is the working mathematical model.

The working mathematical model of this object of research was mainly developed using only one of the principles from [6], the principle of gradual complication. This makes this example similar to the "hierarchical approach to the construction of models" from [3].

\section{Conclusion}

Thus, the concept of the working mathematical model has been formulated. We have also discussed the features of construction of mathematical models with the desired properties from the point of view of a particular study. The sample working mathematical model that has been discussed was mainly developed using only the principle of gradual complication.

\section{References:}

1. Dym CL (2004) Principles of Mathematical Modeling. Elsevier Academic Press.

2. Myshkis AD (2014) Elements of the Theory of Mathematical Models [in Russian]. LENAND, Moscow.

3. Samarskii AA, Mikhailov AP (2002) Principles of Mathematical Modeling: Ideas, Methods, Examples. Taylor \& Francis, London.

4. Velten K (2010) Mathematical Modeling and Simulation: Introduction for Scientists and Engineers. Wiley-VCH-Verl., Weinheim.

5. Zarubin VS (2010) Mathematical Modeling in Engineering [in Russian]. Izd-vo MGTU im. N.E. Baumana, Moscow.

6. Markelov GE (2006) Principles to Construct Mathematical Models. Proc. of the Int. Conf. "Tikhonov and Contemporary Mathematics: Mathematical Modeling", Izd. otd. fak. VMiK MGU im. M.V. Lomonosova, Moscow, pp. 128-129.
7. Markelov GE (2012) Peculiarities of Construction of Mathematical Models. Inzhenernyi zhurnal: nauka i innovatsii, No. 4, Available:

http://engjournal.ru/catalog/mathmodel/hidden/ 150.html (Accessed: 03.11.2012).

8. Markelov GE (2000) Effect of initial heating of the jet-forming layer of shaped-charge liners on the ultimate elongation of jet elements. J. Appl. Mech. and Tech. Phys., 41, No. 2, pp. 231-234.

9. Markelov GE (2000) Effect of initial heating of shaped charge liners on shaped charge penetration. J. Appl. Mech. and Tech. Phys., 41, No. 5, pp. 788-791.

10. Markelov GE (2000) Influence of heating temperature on the ultimate elongation of shaped-charge jet elements. Proc. of the 5th Int. Conf. "Lavrentyev Readings on Mathematics, Mechanics and Physics", Lavrentyev Institute of Hydrodynamics, Novosibirsk, pp. 170. 